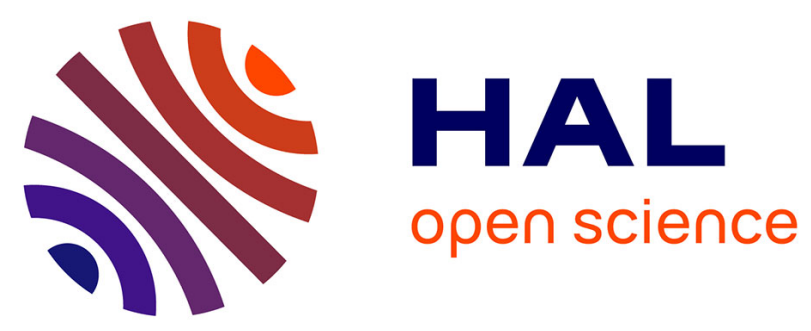

\title{
Training sequence versus cyclic prefix-a new look on single carrier communication
}

Luc Deneire, Bert Gyselinckx, M.G.E. Engels

\section{To cite this version:}

Luc Deneire, Bert Gyselinckx, M.G.E. Engels. Training sequence versus cyclic prefix-a new look on single carrier communication. IEEE Communications Letters, 2001, 5 (7), pp.292-294. 10.1109/4234.935746 . hal-00187743

\section{HAL Id: hal-00187743 https://hal.science/hal-00187743}

Submitted on 15 Nov 2007

HAL is a multi-disciplinary open access archive for the deposit and dissemination of scientific research documents, whether they are published or not. The documents may come from teaching and research institutions in France or abroad, or from public or private research centers.
L'archive ouverte pluridisciplinaire HAL, est destinée au dépôt et à la diffusion de documents scientifiques de niveau recherche, publiés ou non, émanant des établissements d'enseignement et de recherche français ou étrangers, des laboratoires publics ou privés. 


\title{
Training Sequence versus Cyclic Prefix- A New Look on Single Carrier Communication
}

\author{
Luc Deneire, Member, IEEE, Bert Gyselinckx, and Marc Engels, Member, IEEE
}

\begin{abstract}
Orthogonal frequency-division multiplexing (OFDM), with the help of a cyclic prefix, enables low complexity frequency domain equalization, but suffers from a high crest factor. Single carrier with cyclic prefix (SC-CP) has the same advantage with similar performance, but with a lower crest factor and enhanced robustness to phase noise. The cyclic prefix is overhead, so we put more information in it by implementing this cyclic prefix as a training sequence (TS). This new Training Aided frequency domain equalized single carrier (TASC) scheme offers us additional known symbols and enables better synchronization and (potentially) channel estimation, with the same performance as SC-CP.
\end{abstract}

Index Terms-Frequency division multiplexing, synchronization.

\section{INTRODUCTION}

$\mathbf{O}$ NE OF THE major advantages of OFDM is its capability of using low complexity frequency domain equalization (one complex multiplication per data symbol). This is done at the expense of a "Cyclic Prefix," which is able to cope with time dispersive channels, as long as the length of their impulse response is shorter than the cyclic prefix.

The same approach has been adapted to single carrier communications [2], using also a cyclic prefix to absorb the channel. The main advantages of SC versus OFDM are its low crest factor (constant modulus is even achievable by using MSK or constant modulus trellis-coded modulation [3]), and its reduced sensitivity to phase noise [4]. The complexity penalty is that it uses one extra FFT at the receiver side.

The overhead induced by the cyclic prefix can be used in a more efficient way if its content is better known. Indeed, the $\mathrm{CP}$ is hardly useful for channel estimation, and is sometimes insufficient for proper synchronization. We propose to transform the cyclic prefix in a training sequence. This training sequence plays the same role as the cyclic prefix, but at the same time serves as a sequence of pilot symbols for synchronization (carrier synchronization and time offset estimation) and (potentially) channel estimation. The big advantage of this scheme is that pilot symbols are not needed anymore to track synchronization and channel variations. Furthermore, there is seldom any net data rate penalty, some gain (a fraction of a $\mathrm{dB}$ ) can even be achieved in practical situations.

In this letter, we first explain how to modify the framing and data processing to see a training sequence as a cyclic prefix and

\footnotetext{
Manuscript received January 9, 2001. The associate editor coordinating the review of this letter and approving it for publication was Prof. J. Ritcey.

The authors are with IMEC, B-3001 Leuven, Belgium (e-mail: deneire@imec.be).

Publisher Item Identifier S 1089-7798(01)06475-4.
}

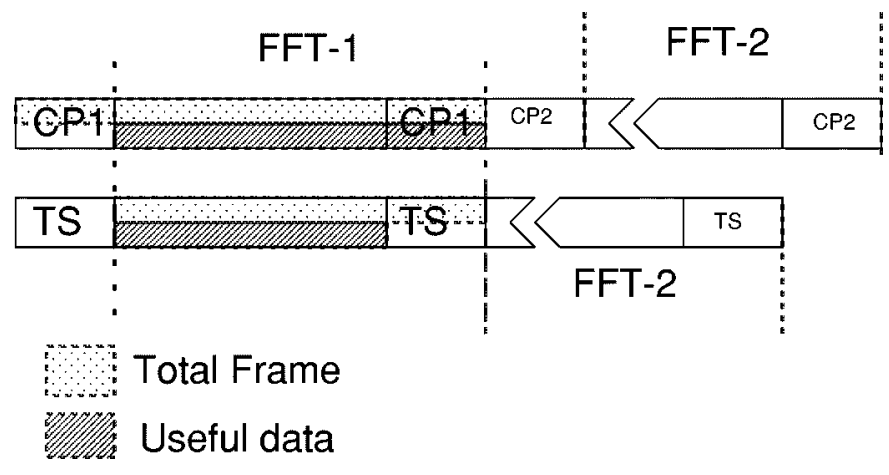

Fig. 1. Single carrier with cyclic prefix and training sequence.

how to use it for synchronization and channel estimation. We show that there is no penalty in performance neither in data throughput and, for enhancing synchronization, propose a maximum-likelihood (ML) algorithm joint estimation of frequency offset and frame timing which can be seen as a generalization of [5]. These arguments show that the proposed TASC is a good candidate for frequency domain equalized single carrier communication schemes.

\section{Cyclic PrefiX as a Training Sequence}

The single carrier counterpart of OFDM consists in sending data frames where the start and end of the frames contain the same data, viz. the cyclic prefix. By doing this, the convolution of the data with the channel becomes a cyclic convolution, and frequency domain equalization is possible with only one multiplication per data symbol (or one tap per subcarrier in the OFDM terminology). The performance of this scheme (taking coding into account) is essentially the same as for OFDM [6], but with enhanced robustness to nonlinear distortion and phase noise [4].

The basic idea of TASC is to transform the cyclic prefix in a training sequence. This TS should be viewed as a cyclic prefix by the FFT device and be always the same and hence, not include useful data. The modification in the framing is depicted in Fig. 1, where the original SC-CP scheme is drawn for comparison. The difference is that, instead of having to throw away the cyclic prefix, we always process the training sequence, hence, there is no gap anymore between two FFT's.

In the OFDM scheme, we note respectively $N_{c}, N_{p}$ and $N_{l}$ as the total number of carriers, the length of the $\mathrm{CP}$ and the number of pilot carriers. In the SC-CP scheme, for the same FFT size, $N_{c}+N_{p}$ is the total number of data symbols, while 


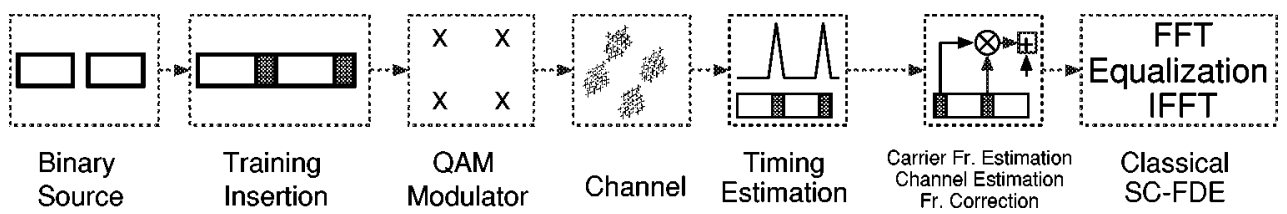

Fig. 2. TASC transmit/receive chain.

$N_{c}-N_{l}$ is the number of useful data symbols, considering an equivalent pilot symbol assisted modulation (PSAM) scheme as for the OFDM case. In the TASC scheme, still for the same FFT size, $N_{c}$ is the total number of data symbols, while $N_{c}-N_{p}$ is the number of useful data symbols, $N_{p}$ being the length of the TS. To compare overheads, one can compute the ratio of the number useful data symbols versus the number of total data symbols, which is $\left(N_{c}-N_{p} / N_{c}\right)$ for TASC and $\left(N_{c}-N_{l} / N_{c}+\right.$ $N_{p}$ ) for SC-CP and OFDM, leading to a gain in data rate of $\left(N_{c}^{2}-N_{p}^{2} / N_{c}^{2}-N_{c} N_{l}\right)$ for the TASC scheme, compared to SC-CP and OFDM. Obviously, if $N_{c} N_{l}>N_{p}^{2}$, there is a gain in net data rate (and loss if not). As an example, for $N_{l}=4, N_{p}=$ 16 and $N_{c}=64$, there is no modification in the data rate, for $N_{l}=4, N_{p}=16$ and $N_{c}=256$, the gain is $0.05 \mathrm{~dB}$ and for $N_{l}=8, N_{p}=16$ and $N_{c}=256$, the gain is $0.12 \mathrm{~dB}$. Compared to classical single carrier, the overhead can be made similar by an appropriate choice of the parameters.

From a performance point of view, the TASC scheme inherits from the properties of the SC-CP scheme: it offers a similar performance as for OFDM, with more robustness to nonlinear distortion (lower crest factor) and phase noise.

The global Transmit/Receive chain becomes as in Fig. 2. Before diving into our sample algorithm taking advantage of the short training sequence that is provided, let's see what we can expect a priori. The TS sequence does not contain data. Hence, it can be optimized to get appropriate properties (e.g., autocorrelation) and its symbols could even be chosen from a separate alphabet. This avoids the accidental presence of the TS sequence in the useful data, which makes the TASC akin to PSAM (Pilot-symbol-assisted modulation). A scheme inspired by [7] could for example be adopted for frame synchronization.

From a synchronization point of view, the TASC acquisition is essentially the same as for the SC-CP: as the TS sequence is rather short, we can probably not rely on it for this task. For the tracking algorithms, data-aided (DA) algorithms are known to perform better than their non-data-aided counterparts (NDA) (see [8, Ch. 6]). They avoid decision directed algorithms and alleviate the problem of feeding the decisions back, which would mean a delay of one frame in the SC-CP and TASC schemes.

For the channel estimation, the concept of semi-blind identification [9] (i.e., using both the TS sequence and the statistical properties of the signals) can be applied directly here, with possible extensions to the multiuser case. Of course, these methods are most useful when the channel is varying rather rapidly, like in mobile communications.

The extension of TASC to the multiuser case (in a spatial division multiple access scheme) is easier than for SC-CP, as the users can be distinguished on the basis of their (different) TS.

\section{JoINT TIME/FREQUENCY SYNCHRONIZATION}

Based on an extended observation time, we introduce the joint ML estimators for the frequency and delay.

We base ourselves on an observation time incorporating $M(M \geq 2)$ training sequences, without using the knowledge of this TS (hence using only the periodicity property of the TS). The reason for this choice is twofold: on one hand, use of both knowledge of the TS and the periodicity of the TS would lead to complex algorithms, on the other hand, in practical systems, acquisition of synchronization will be based on specific preambles, hence the additional performance expected by use of the knowledge of the TS will not be critical (indeed, the performance improvement shown below is sufficient to yield good synchronization).

A two-step algorithm gives us the ML estimator, extending [5] to the multidimensional case. Denoting $\tau$ as the delay between the start of the measurement interval and the start of the data frame, $\sigma_{x}^{2}$ as the power of the signal at the output of the channel, $\sigma_{n}^{2}$ as the noise power and $y(t)=x(t)+n(t)$ the output of the channel, the first step gives us the ML estimator of the delay $\hat{\tau}_{\mathrm{ML}}$

$$
\hat{\tau}_{\mathrm{ML}}=\arg \max _{\tau} \operatorname{trace}\left\{\mathcal{Y} \mathcal{Y}^{H}\right\}-M \frac{\sigma_{x}^{2}}{\sigma_{x}^{2}+\sigma_{n}^{2}} \mathbf{1}^{H}\left|\mathcal{Y} \mathcal{Y}^{H}\right| \mathbf{1}
$$

where

$$
\mathcal{Y}=\left[\begin{array}{ccc}
y(\tau) & \cdots & y\left(\tau+N_{p}-1\right) \\
y\left(\tau+N_{c}\right) & \cdots & y\left(\tau+N_{c}+N_{p}-1\right) \\
\vdots & \ddots & \vdots \\
y\left(\tau+(M-1) N_{c}\right) & \cdots & y\left(\tau+(M-1) N_{c}+N_{p}-1\right)
\end{array}\right]
$$

and 1 is a column vector of ones.

In the second step, $\widehat{\Delta f} f_{\mathrm{ML}}$ can be computed from

$$
W\left(\widehat{\Delta f}_{\mathrm{ML}}\right)=V_{\max }\left\{\mathcal{Y}\left(\hat{\tau}_{\mathrm{ML}}\right) \mathcal{Y}\left(\hat{\tau}_{\mathrm{ML}}\right)^{H}\right\},
$$

where $V_{\max }\{A\}$ denotes the eigenvector corresponding to the maximum eigenvalue of matrix $A$ and $W(\Delta f)=$ $\left[1 e^{-22 \pi N_{c} \Delta f} \cdots e^{-22 \pi N_{c}(M-1) \Delta f}\right]^{H}$.

Note that, when synchronized and in the absence of noise, $\mathcal{Y}$ is of rank one (all lines are equal up to a phase), hence $\mathcal{Y} \mathcal{Y}^{H}$ is of rank one, which is an interesting property for the tracking.

From a complexity point of view, even if the eigenvalue decomposition can appear frightening, the simulations show that we get most of the benefits for small values of $M$ (4 to 6), hence, the eigenvalue decomposition has only to be performed on small $M \times M$ matrices. Furthermore, the structure of the matrix is such that it can be updated iteratively, as well as the decomposition, if bigger values of $M$ are used. The computation of the matrix itself can be done by simple correlation devices. 


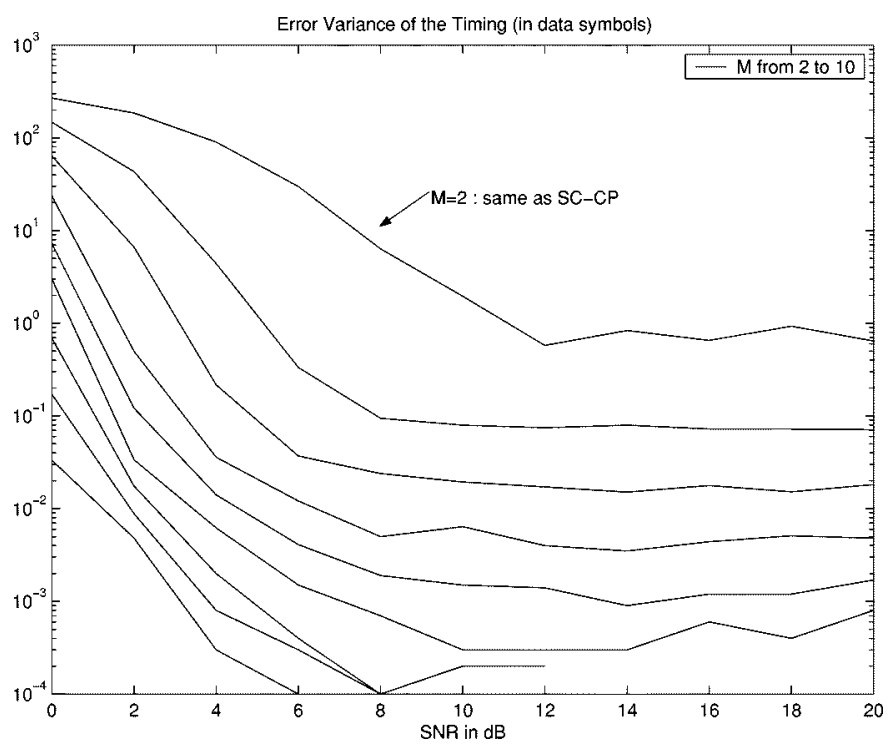

Fig. 3. Performance of the frame synchronization is improved a lot for low SNR and a small increase in observation time.

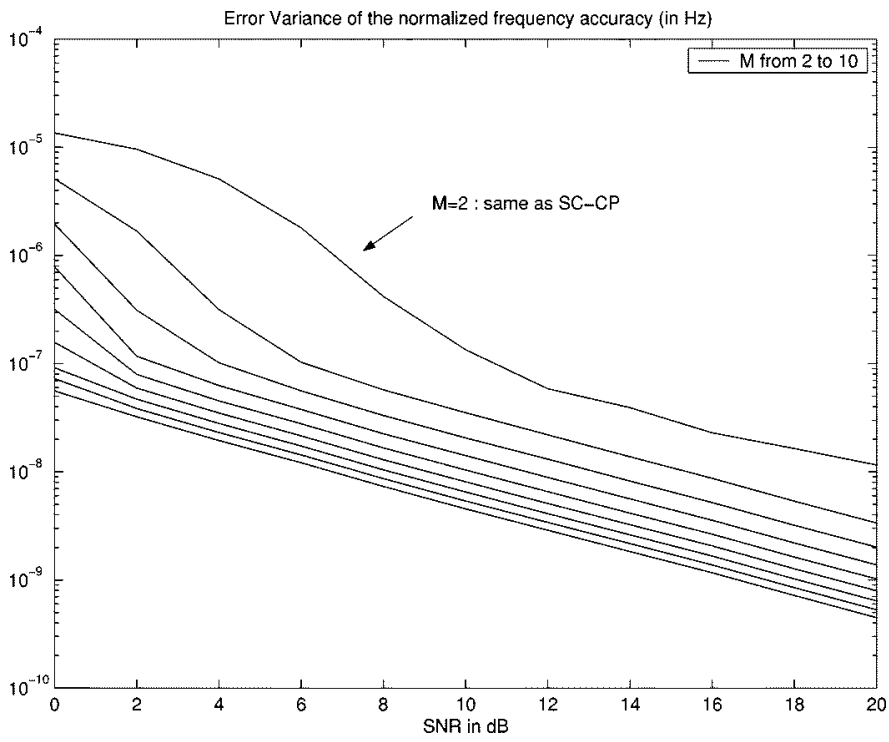

Fig. 4. Performance of the frequency synchronization is improved a lot for low SNR and a small increase in observation time.

We have performed simulations with 10000 frames consisting of $N_{c}=64$ QPSK symbols, among which there are $N_{p}=8$ training symbols. The algorithm has been run for SNR's from 0 to $20 \mathrm{~dB}$ and using from 2 to $10(M=2 \cdots 10)$ training sequences in the observation interval. The performances in Figs. 3 and 4 have to be compared to the upper curve (for $M=2$ ), which corresponds to the ML solution for the $\mathrm{SC}-\mathrm{CP}$ solution. The benefit of the modified scheme is evident for short observation intervals at low SNR's. It really extends the framing synchronization capabilities to the low SNR's and offers a good improvement on the frequency synchronization.

\section{CONCLUSIONS}

We have introduced a new TASC scheme taking advantage of the cyclic prefix nature of a short training sequence. This allows to perform frequency domain equalization at low computational complexity and to improve the performance of the synchronization by a rather simple algorithm. To this end, we have derived the ML joint estimation of frequency offset and frame timing, based on a model relying only on the cyclic repetition of the training sequence, for an AWGN channel. Simulations show a significant improvement on timing estimation at low SNRs and for a small amount of frames.

Further incorporation of the knowledge of the training sequence itself should lead to another bunch of synchronization algorithms and should also benefit from the extensive literature on sequence design for synchronization. Moreover, this TS can also be used to perform semi-blind channel identification, which is useful in fast fading channels (like in mobile communications), and easily applicable to the multiuser case.

These considerations make TASC an interesting candidate in situations where multipath and fast fading are present, while in other situations it will perform like SC-CP.

\section{REFERENCES}

[1] A. Czylwik, "Low overhead pilot-aided synchronization for single carrier modulation with frequency domain equalization," in GLOBECOM, vol. 4, 1998, pp. 2068-73.

[2] H. Sari, G. Karam, and I. Jeanclaude, "Frequency-domain equalization of mobile radio and terrestrial broadcast channels," in GLOBECOM, 1994, pp. 1-5.

[3] H. Sari, G. Karam, V. Paxal, and K. Maalej, "Trellis-coded constantenvelope modulations with linear receivers," IEEE Trans. Commun., vol. 44, pp. 1298-1307, Oct. 1996.

[4] T. Pollet, M. V. Bladel, and M. Moeneclaey, "BER sensitivity of OFDM systems to carrier frequency offset and wiener phase noise," IEEE Trans. Commun., vol. 43, pp. 191-193, Feb.-Apr. 1995.

[5] J. V. de Beek, M. Sandell, and P. Börjesson, "Ml estimation of time and frequency offset in OFDM systems," IEEE Trans. Signal Processing, vol. 45, pp. 1800-1805, July 1997.

[6] H. Sari, "Channel equalization and carrier synchronization issues in multicarrier transmission," in IEEE Synchronization Workshop, Belgium, pp. 29-36.

[7] J. A. Gansman, M. P. Fitz, and J. V. Krogmeier, "Optimum and suboptimum frame synchronization for pilot-symbol-assisted modulation," IEEE Trans. Commun., vol. 45, pp. 1327-1337, Oct. 1997.

[8] H. Meyr, M. Moeneclaey, and S. A. Fletchel, Digital Communication Receivers: Synchronization, Channel Estimation and Signal Processing: Wiley, 1997.

[9] E. de Carvalho and D. Slock, "Cramer-Rao bounds for semi-blind, blind and training sequence based channel estimation," in First IEEE Signal Processing Workshop on Signal Processing Advances in Wireless Communications, 1997, pp. 129-132. 